\title{
Research on Data Optimization of CT System for Patients with Severe Pneumonia Based on Filtered Back Projection and Internet of Things
}

\author{
Shaojie Qin and Wentao Wang \\ Intensive Care Unit of The Second Affiliated Hospital, Zhengzhou University, Zhengzhou 450003, China \\ Correspondence should be addressed to Wentao Wang; zzdxdff@zzu.edu.cn
}

Received 17 January 2021; Revised 9 February 2021; Accepted 24 February 2021; Published 13 March 2021

Academic Editor: Chi-Hua Chen

Copyright (C) 2021 Shaojie Qin and Wentao Wang. This is an open access article distributed under the Creative Commons Attribution License, which permits unrestricted use, distribution, and reproduction in any medium, provided the original work is properly cited.

\begin{abstract}
CT technology can clearly and intuitively display the internal structure, composition, material, and defect status of the detected object under the condition of no damage to the detected object. This paper is based on the filtering back projection and the Internet of Things technology to carry out the data optimization of the CT system of patients with severe pneumonia. First of all, the Internet of Things technology is used in the structural design and operation process of the CT system for patients with severe pneumonia, which improves the advancement and convenience of the original system. Secondly, the system data is optimized through the filtering back projection algorithm, which improves the data accuracy and transmission efficiency. Finally, simulation tests have verified the effectiveness of the established CT system data optimization method for patients with severe pneumonia, which is helpful for medical comparison, analysis, and diagnosis.
\end{abstract}

\section{Introduction}

Computed tomography (CT) is a medical diagnosis method that combines electronic computer technology and X-ray imaging technology. Due to the rapid development of electronic computer technology in recent years, this method has become one of the most common medical clinical diagnostic methods $[1,2]$. Severe pneumonia is a common respiratory disease. The various organs of the elderly are gradually failing, the immune system is weakened, and they are more likely to suffer from severe pneumonia [3]. The highresolution CT image can accurately detect the subtle differences in the radiation attenuation characteristics between the tissues on the cross-section of the human body to be measured and display them in the form of digital images and can finely distinguish the different densities [4]. Therefore, CT technology is widely used in cross-sectional tissues for medical purposes [5]. The disease has the characteristics of insidious onset, critical illness, and rapid progress. The initial symptoms are local inflammation gradually worsening to systemic inflammation, causing severe infectious compli- cations such as septic shock, sepsis, and multiple organ dysfunction, which poses a great threat to the life safety of patients and requires effective treatment intervention as soon as possible $[6,7]$.

Although the above-mentioned medical imaging modes have a wide range of important applications, due to factors such as the performance of their own equipment and external conditions, medical images still have many unavoidable defects $[8,9]$. CT technology can clearly and intuitively display the internal structure, composition, material, and defect status of the inspected object without damaging the inspected object $[10,11]$, for example, the image is blurred or the image boundary is not obvious. In addition, in the process of forming and transmitting medical images, its image quality will also be affected to a certain extent [12]. All these have caused certain difficulties and obstacles to doctors' image interpretation and accurate diagnosis. In order to enhance the quality of medical images, enable doctors to observe and analyze the lesions and anatomical structures more clearly, and improve the accuracy of diagnosis, postprocessing of medical images becomes necessary 
$[13,14]$. This paper is based on the filtering back projection and the Internet of Things technology to carry out the data optimization of the CT system of patients with severe pneumonia. The established CT system data optimization method for patients with severe pneumonia is helpful for medical comparison, analysis, and diagnosis.

\section{CT Tomography Technology}

CT is computed tomography. CT imaging uses the attenuation characteristics of X-rays as a diagnostic basis to scan through a certain thickness of a certain part of the human body $[14,15]$. The high-sensitivity receiver receives the $\mathrm{X}$ rays that pass through this layer and converts them into visible light, which is converted into electrical signals by photoelectric conversion, and then converted into digital signals by an analog-digital converter. Each number in the digital matrix is converted into small squares from black to white (i.e., pixels) by a digital-to-analog converter and arranged into a matrix to obtain CT images $[16,17]$. After entering the computer, the selected layer is divided into multiple voxels, and the scanned information is calculated to obtain the X-ray attenuation coefficient or absorption coefficient of each voxel and arranged in a digital matrix.

$\mathrm{X}$-rays have varying degrees of penetrating ability to various substances. When a beam of X-rays passes through the human body, it will be attenuated due to absorption and scattering $[18,19]$. However, due to the different density and atomic composition of various tissues of the human body, the degree of absorption and scattering is also different, that is, different tissues of the human body have different attenuation coefficients for X-rays [20, 21]. In general, when $\mathrm{X}$-rays with the same energy are used for incidence, the higher the density of human tissues, the greater the attenuation of X-rays, and the lower the density of human tissues, the lower the attenuation of $\mathrm{X}$ rays. CT images express the absorption degree of X-rays by organs and tissues in different gray scales, which is consistent with X-ray imaging. The black areas indicate low absorption areas, i.e., low-density areas, such as lungs; white areas indicate high absorption areas, i.e., high-density areas $[22,23]$.

However, compared with X-ray images, CT is completely dependent on the projection data of the attenuation value of the object in the inspected layer. It has nothing to do with the structure of the inspected layer. It is a complete tomographic image with more accurate layer thickness and clearer image. The density resolution is higher. In addition, the tomographic images obtained by CT scanning can also be processed by computer software to obtain multidirectional cross-sectional images, such as coronal images and sagittal images, for easy observation [24]. The above characteristics provide an important guarantee for the research to obtain clear, high-resolution, and economical geometric information of cerebral aneurysms. The reconstructed image can be displayed on the monitor in different Di levels or stored in the computer hard disk in digital form.

\section{Overview of IoT Technology}

The Internet of Things has entered all aspects of people's lives, and as a technical support for economic development, it has shown great potential as a promoter of world economic growth in the 21 st century $[25,26]$. The Internet of Things is a systematic fusion of multiple technologies. To understand the Internet of Things, we should start from its system architecture and fully understand it to grasp the overall situation from the top level and lay a solid foundation for specific application design $[27,28]$. A sensor conduction network is composed of probes, remote sensing components, and various terminal devices $[29,30]$. The sensing layer is composed of different forms of sensing components, QR codes, RFID tags, and barcode readers distributed in various building groups. A schematic diagram of IoT technology architecture is shown in Figure 1.

The access network is a fusion of the communication network and the Internet composed of various wired and wireless nodes and fixed and mobile gateways and can be accessed through the Internet, the broadcasting network, and the communication network. Different from classic information systems, IoT requires large-scale data access and transmission capabilities [31-33]. The role of the middleware layer is to shield heterogeneity, achieve two-way interaction, and data preprocessing. At the same time, various system platforms and technical resources with different advantages are integrated and shared to form a multifunctional integrated system with diverse resource allocation and complete functions. The middleware layer can provide a variety of services to the upper layer, integrate multiple applications on the basic system, and provide differentiated services for different user needs.

\section{Optimization of CT System Data for Patients with Severe Pneumonia}

4.1. Filtering Back Projection Algorithm. Interpolation here refers to a model-based method used to estimate continuous data from discrete data within a known coordinate range. Classical linear interpolation techniques include nearest neighbor interpolation, bilinear interpolation, and bicubic interpolation $[34,35]$. In the back-projection process of the FDK algorithm, due to the discreteness of the data, the projection address of the pixel will appear "misaligned," which usually requires interpolation, especially for the edges of the image. The nearest neighbor interpolation will produce blackness and jaggedness, while bilinear interpolation and bicubic interpolation will blur the edges of the image. Therefore, researchers have introduced many nonlinear interpolation methods, such as local adaptive interpolation methods.

In an ideal situation, the projection is the integral of the specific parameters of the object along a straight line passing through the object, and the measured object is expressed as a two-dimensional function, that is, the angle between the ray normal and the light.

$$
\rho=x \cos \theta+y \sin \theta \text {. }
$$




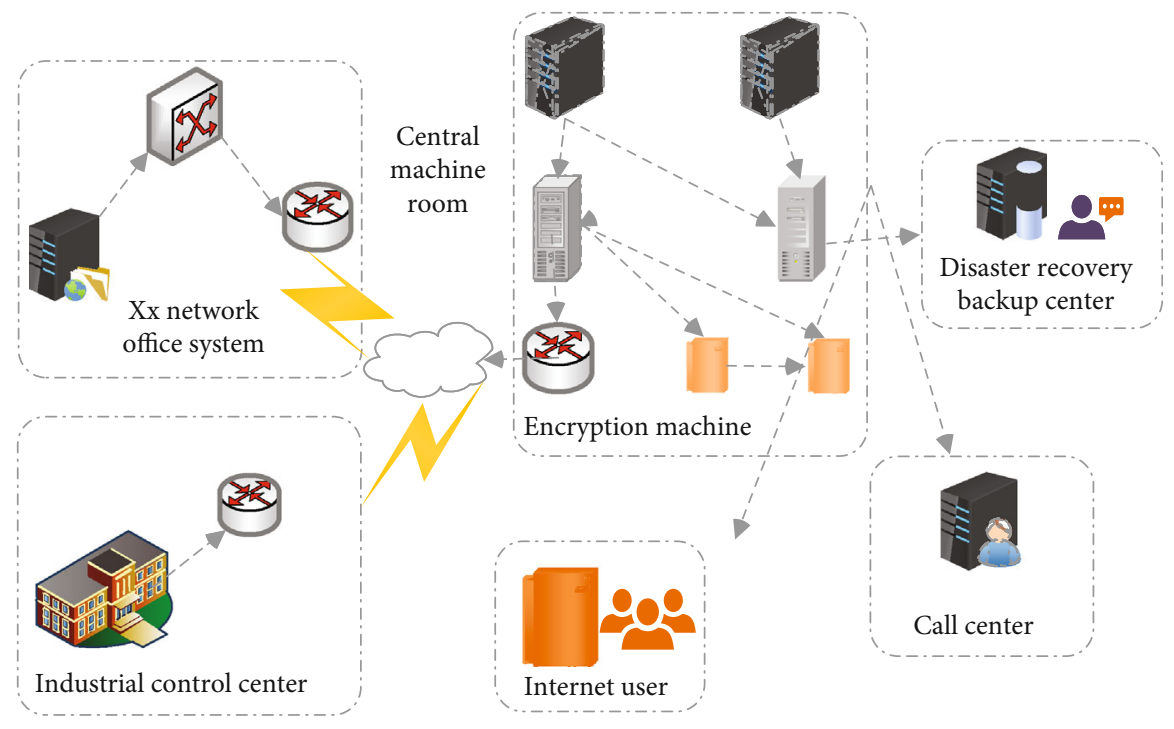

FIgURE 1: Schematic diagram of IoT technology architecture.

According to the conversion relationship between polar coordinates and coordinates, $\beta=\arctan (y / x)$ and $r=x^{2}+$ $y^{2}$, the formula can be rewritten as

$$
\rho=r \cos (\theta-\beta) .
$$

A schematic diagram of the filtered projection geometric ray is shown in Figure 2. According to the geometric properties of the parallel projection, after the ray scan, the complete image of the original image should be obtained, and a series of projection data are measured [36]. The projections of all rays passing through a certain pixel are accumulated in the projection domain. This pixel can be reconstructed:

$$
f(x, y)=\int_{0}^{\pi} G(x \cos \theta+y \sin \theta, \theta) d \theta .
$$

The geometric relationship between these pixels is shown in the figure. According to the geometric relationship, the angle between the ray and the pixel is

$$
f(r, \alpha)=\int_{0}^{\pi} G(r \cos \varphi, \theta) d \theta .
$$

A schematic diagram of the filtered projection polar coordinate geometry is shown in Figure 3. It can be seen from the above formula that the projection length is no longer dependent on this angle, so the area to be solved for the image is transformed from the plane with $X Y$ as the horizontal and vertical coordinates to the polar coordinate plane with the length as the rotation angle [37]. In the process of CT image reconstruction, it is first converted from the $X Y$ coordinate domain to the polar coordinate domain and then reconstructed [38].

4.2. Optimization of CT System Data for Patients with Severe Pneumonia Based on Filtered Back Projection. What is obtained after reconstructing the image in the polar coordi-

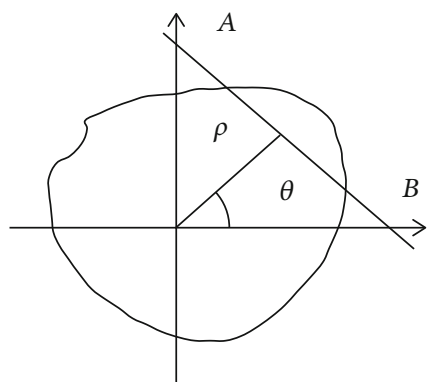

Figure 2: Schematic diagram of filtered projection geometric ray.

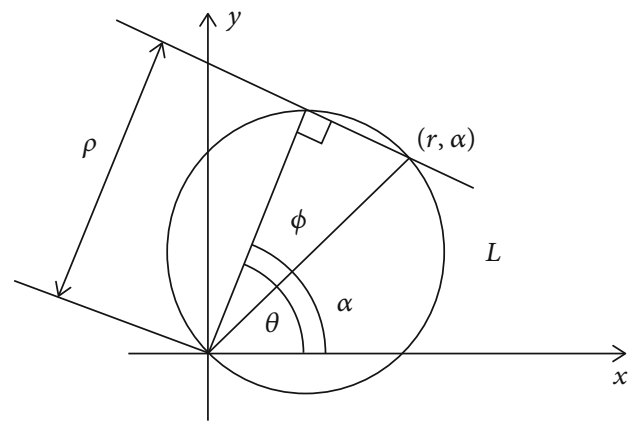

FIGURE 3: Schematic diagram of filtered projection polar coordinate geometry.

nate domain is not the final result. It is necessary to use the coordinate conversion relationship $x=r \cos \alpha$ and $y=r \sin$ $\alpha$ and map it to the $X Y$ coordinate system to truly reflect the original image. MATLAB software is used to run the program to get the image shown in Figure 4.

In this paper, after processing the absorption matrix with MATLAB, the absorption rate image of the object can be obtained, as shown in Figure 1. The figure shows that the object is uniform. When X-rays penetrate an object, the rays 


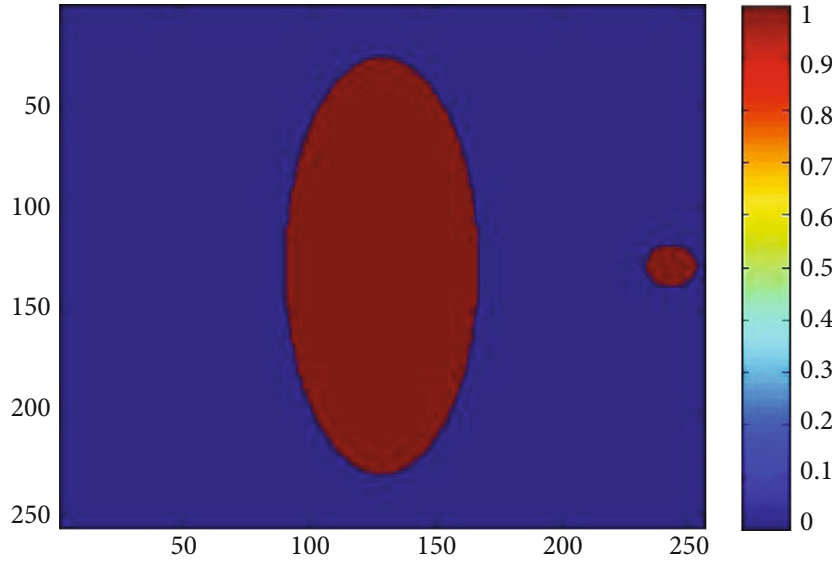

FIGURE 4: The geometry of the filtered projection medium.

will be absorbed and scattered by the material, which will weaken the intensity of the X-rays. It can be known from Beer's theorem that when a single light is emitted, the attenuation of the X-ray of the object in a single uniform material will change, and the attenuation of its intensity satisfies formula (1).

$$
I=I_{0} e^{-\mu \Delta x}
$$

Assuming that the test object is segmented and uniform, the linear attenuation coefficients of each segment are as shown in formula (2):

$$
\mu_{1} x_{1}+\mu_{2} x_{2}+\mu_{3} x_{3}+\cdots=\ln \left(\frac{I_{0}}{I}\right)
$$

Take an ideal ray source, which emits extremely thin pen beam rays, and place a detector on the opposite side. Measure the ray receiving signal $g$ and the intensity of the rays reaching the detector after being attenuated by the object, thus obtaining a set of data. Then, rotate at an angle to obtain another set of data under the new angle. Repeat this until 180 sets of data are obtained after 180 rotations. The fitting diagram of the filtered projection received information data is shown in Figure 5.

According to Radon transformation, suppose the absorption function of a beam of parallel light is shown in formula (3):

$$
f(x, y)\left\{\begin{array}{cc}
\neq 0, & (x, y) \in \Omega, \\
=0, & (x, y) \notin \Omega .
\end{array}\right.
$$

Among them, $\Omega$ is the medium, and the attenuation rate of the intensity is $d I / I=-f(x, y) d u$. Formula (4) is solved:

$$
I=I_{0} \exp \left[-\int_{L} f(x, y) d u\right] .
$$

The relationship between the coordinate system $X-Y$

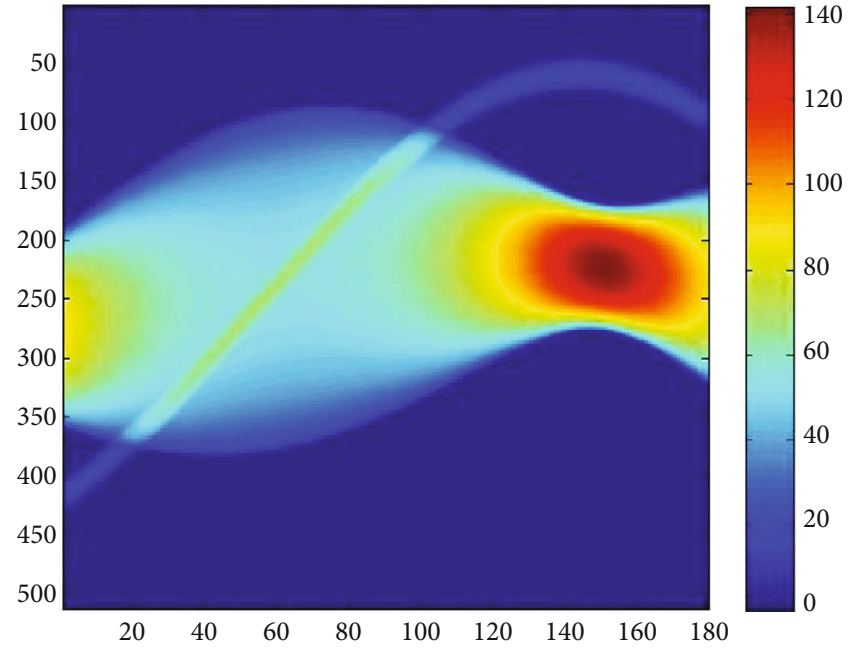

FIgURE 5: Fitting diagram of filtered projection received information data.

established with the pallet as the reference system and the coordinate system $s$ - $u$ established with the detector as the reference system is shown in

$$
\left\{\begin{array}{l}
s=X \cos \theta+Y \sin \theta, \\
u=-X \sin \theta+Y \cos \theta .
\end{array}\right.
$$

From the data fitting diagram of the received information of the template, it can be seen that the curve in the figure is approximately fitted to a sine function. According to the symmetry of the sine function, the abscissa of the origin can be calculated as 62 , and the abscissa of the lowest point of the sine function is 144 . Corresponding to the distance between the abscissas of the two points, the radian corresponding to the entire curve can be calculated, that is, the entire CT system rotates counterclockwise around the center of rotation, and a total of 180 sets of received information are obtained. It can be calculated that the CT system receives information every time it passes.

$$
\begin{gathered}
g(s, \theta)=R f=\int_{-\infty}^{+\infty} \int_{-\infty}^{+\infty} f(x, y) \delta(x \cos \theta+y \sin \theta-s) \\
\cdot d x d y, \quad-\infty<s<+\infty, 0 \leq \theta \leq \pi
\end{gathered}
$$

Calculate the expression of the sine function, and then obtain $\phi=3 \pi / 4$, that is, the initial angle is $3 \pi / 4$, so the 180 directions of the $X$-ray used by the CT system are $135^{\circ}+1.0980 N^{\circ}$, where $N$ represents the number of rotations.

\section{Optimization of CT System Data for Patients with Severe Pneumonia}

5.1. Data Source and Test Environment. In order to verify the effectiveness of this method on CT images, a simulated severe pneumonia CT image was used as a test sample, the similarity 


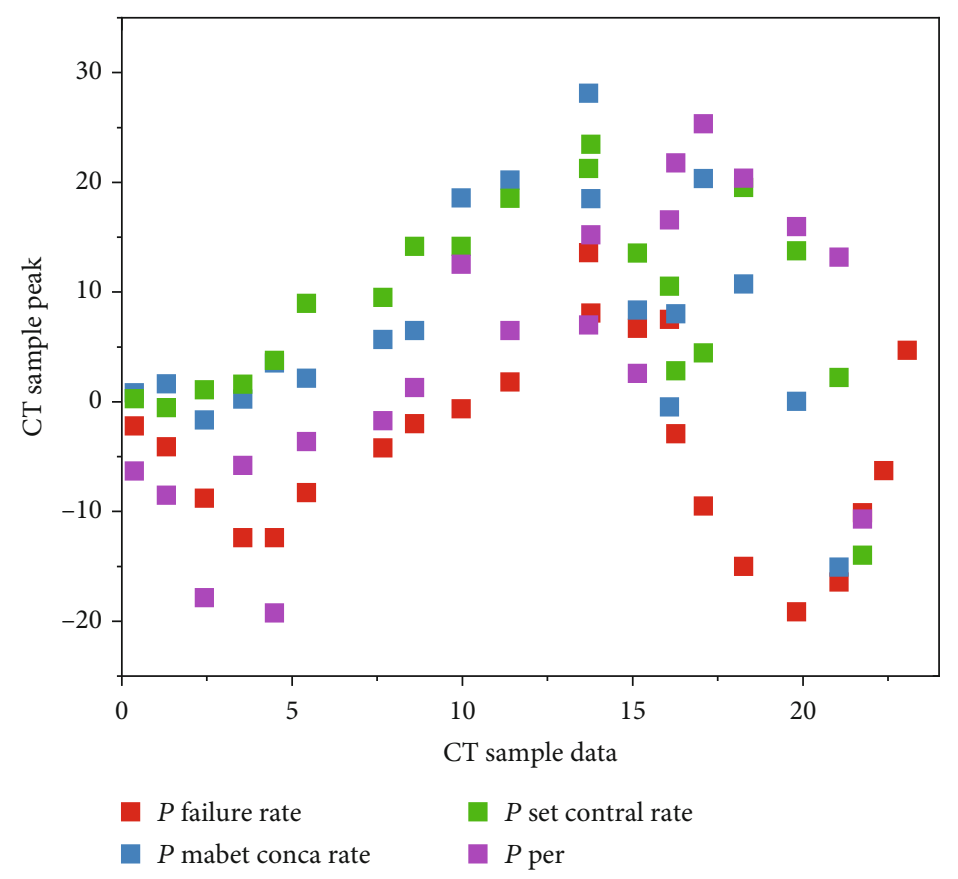

Figure 6: Peak signal-to-noise distribution of CT samples under different conditions.

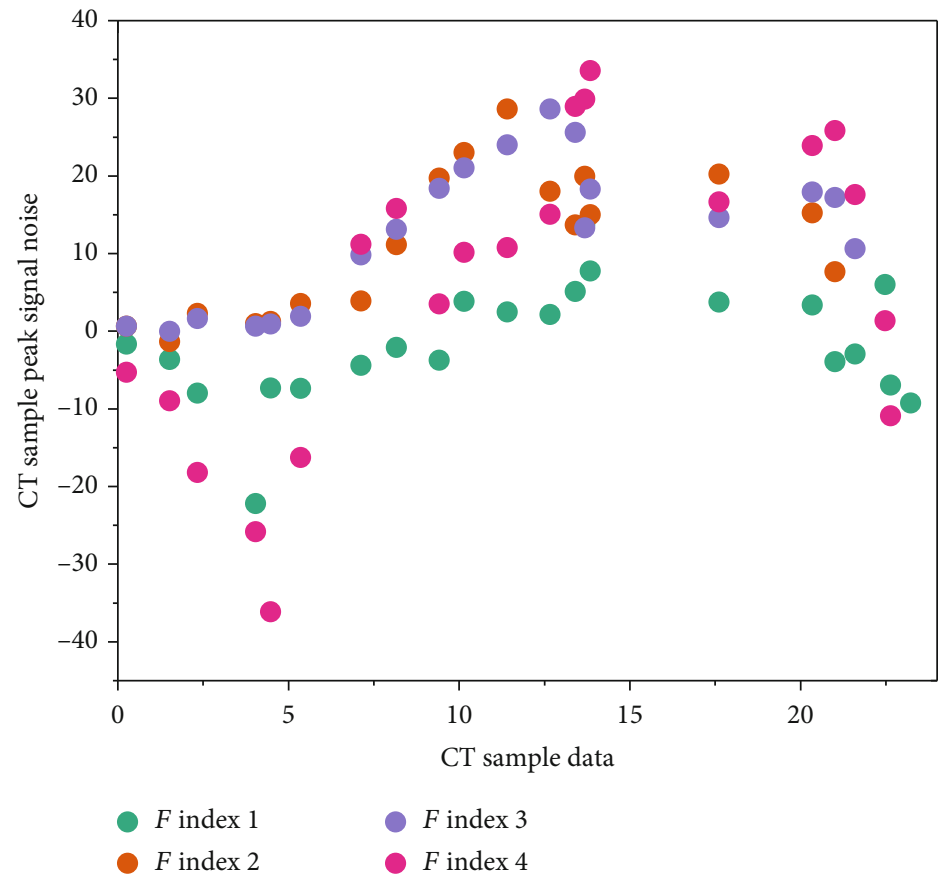

Figure 7: Peak distribution of CT samples under different search window radii.

window radius was selected as 3 , the search window radius was selected as 7, and the smoothing coefficient was selected as 0.2 , and the algorithm comparison experiment was performed.

On the basis of the description of the filtering back projection algorithm, the Radon inverse transformation is performed on the projection image generated by the data again, and the filtering back projection algorithm is established. The sample image is added with Gaussian noise inter- ference with a mean value of 0 and a variance of 0.01 before proceeding. In terms of noise processing, we use the filtered back projection algorithm, the DCT self-similar algorithm and the DCT threshold de-coefficient self-similar algorithm. The result of comparing the denoising effect is shown in Figure 6, which compares the peak signal-to-noise ratio and the signal-to-noise ratio. In addition, the experimental results of the three result images are shown in Figure 7. After acquiring a digital image, the first thing to do is to preprocess the 
image to improve the image quality or reduce the amount of calculation. The specific content of image preprocessing depends on the specific system requirements. Edge extraction first detects the discontinuities of the local characteristics of the image and then connects these discontinuous edge pixels into a complete boundary. In the image, the areas where the gray level changes drastically, that is, the intensity discontinuity, correspond to the edges. Edge extraction is to detect the intensity discontinuities and to determine their precise position in the image. It can be seen that the peak signal-tonoise ratio and signal-to-noise ratio of the simulated CT image obtained by the DCT self-similar algorithm and the DCT threshold decoefficient self-similar algorithm are larger than those of the original algorithm. Therefore, through experimental simulation comparison, we believe that the improved filtered back projection algorithm has better results in CT image processing.

\section{Conclusion}

CT technology can clearly and intuitively display the internal structure, composition, material, and defect status of the inspected object without damaging the inspected object. In order to improve the quality of medical images, enable doctors to observe and analyze lesions and anatomical structures more clearly, and improve the accuracy of diagnosis, postprocessing of medical images becomes necessary. Based on the filtering back projection and the Internet of Things technology, this paper optimizes the data of the CT system of patients with severe pneumonia. The established data optimization method of the CT system for severe pneumonia is helpful for medical comparison, analysis, and diagnosis. Although the above-mentioned medical imaging modes have extensive and important applications, medical images still have many unavoidable defects due to factors such as the performance of their own equipment and external conditions. For example, the image is blurred or the image boundary is not obvious. In addition, in the process of forming and transmitting medical images, the image quality will also be affected to a certain extent. We will further carry out research in related fields to improve the data optimization level of the CT system.

\section{Data Availability}

The datasets used and/or analyzed during the current study are available from the corresponding author on reasonable request.

\section{Ethical Approval}

This article does not contain any studies with human participants or animals performed by any of the authors.

\section{Conflicts of Interest}

The authors declare that they have no conflicts of interest.

\section{Authors' Contributions}

All authors agree to submit this version of the manuscript.

\section{References}

[1] Q. Zhu, B. Zhang, Q. Chen et al., "Model reductions for multiscale stochastic optimization of cooling water system equipped with closed wet cooling towers," Chemical Engineering Science, vol. 224, 2020.

[2] T. Zhang, Y. Xing, L. Zhang, X. Jin, H. Gao, and Z. Chen, "Stationary computed tomography with source and detector in linear symmetric geometry: direct filtered backprojection reconstruction," Medical Physics, vol. 47, no. 5, pp. 22222236, 2020.

[3] M. Tozakidou, I. Apine, K. U. Petersen, J. M. Weinrich, and J. Herrmann, "Comparison of different iterative CT reconstruction techniques and filtered back projection for assessment of the medial clavicular epiphysis in forensic age estimation," International Journal of Legal Medicine, vol. 134, no. 1, pp. 431-443, 2019.

[4] O. Hermine, X. Mariette, P. L. Tharaux et al., "Effect of tocilizumab vs usual care in adults hospitalized with COVID-19 and moderate or severe pneumonia: a randomized clinical trial," JAMA Internal Medicine, vol. 181, no. 1, pp. 32-40, 2021.

[5] K. Smart, A. M. Bröske, D. Rüttinger et al., "PK/PD mediated dose optimization of emactuzumab, a CSF1R inhibitor, in patients with advanced solid tumors and diffuse-type tenosynovial giant cell tumor," Clinical Pharmacology \& Therapeutics, vol. 108, no. 3, pp. 616-624, 2020.

[6] J. van Sluis, R. Boellaard, R. A. Dierckx, G. Stormezand, A. W. J. M. Glaudemans, and W. Noordzij, "Image quality and activity optimization in oncologic 18F-FDG PET using the digital biograph vision PET/CT system," Journal of Nuclear Medicine, vol. 61, no. 5, pp. 764-771, 2020.

[7] L. Shahrin, M. J. Chisti, B. Brintz et al., "Clinical and laboratory predictors of 30-day mortality in severe acute malnourished children with severe pneumonia," Tropical Medicine \& International Health, vol. 25, no. 11, pp. 1422-1430, 2020.

[8] M. Schloz, T. C. Pekin, Z. Chen, W. van den Broek, D. A. Muller, and C. T. Koch, "Overcoming information reduced data and experimentally uncertain parameters in ptychography with regularized optimization," Optics Express, vol. 28, no. 19, pp. 28306-28323, 2020.

[9] Y. Qichun, Z. Xuesong, E. James, M. Almendinger, and $\mathrm{X}$. Huang, "Microlocation for smart buildings in the era of the Internet of Things-a survey of technologies, techniques, and approaches," Environmental Pollution, vol. 16, 2019.

[10] C. Michie, I. Andonovic, C. Davison et al., "The Internet of Things enhancing animal welfare and farm operational efficiency," Journal of Dairy Research, vol. 87, no. S1, Supplement 1, pp. 20-27, 2020.

[11] S. N. Matheu, J. L. Hernández-Ramos, A. F. Skarmeta, and G. Baldini, "A survey of cybersecurity certification for the Internet of Things," ACM Computing Surveys, vol. 53, no. 6, pp. 1-36, 2021.

[12] M. Tozakidou, I. Apine, K. U. Petersen et al., "Comparison of different iterative CT reconstruction techniques and filtered back projection for assessment of the medial clavicular epiphysis in forensic age estimation," International Journal of Legal Medicine, vol. 134, no. 1, pp. 355-361, 2020. 
[13] S. Luo, L. Zheng, S. Luo, N. Gu, and X. Tang, "Data sustained misalignment correction in microscopic cone beam CT via optimization under the Grangeat epipolar consistency condition," Medical Physics, vol. 47, no. 2, pp. 498-508, 2020.

[14] J. Liu, H. R. Zhao, H. L. Wei et al., "Efficacy of bronchoalveolar lavage as adjunct therapy in the treatment of neonatal severe pneumonia: a prospective case-control study," Journal of Tropical Pediatrics, vol. 66, no. 5, pp. 528-533, 2020.

[15] F. Liu, L. Lei, L. Haitao, S. Liping, and P. Longkai, “The level of plasm donor-derived cell-free DNA in kidney transplant patients with severe pneumonia," Transplantation, vol. 104, no. S3, p. S317, 2020.

[16] F. Kassem and G. Kang, "Security and privacy in the Internet of Things," Computer, vol. 43, no. 13, pp. 765-778, 2019.

[17] H. Jiang, Z. Xiao, Z. Li, J. Xu, F. Zeng, and D. Wang, “An energy-efficient framework for Internet of Things underlaying heterogeneous small cell networks," IEEE Transactions on Mobile Computing, vol. 14, no. 2, pp. 232-246, 2020.

[18] J. Huang, J. Guo, H. Li, W. Huang, and T. Zhang, "Efficacy and safety of adjunctive corticosteroids therapy for patients with severe community-acquired pneumonia: a systematic review and meta-analysis," Medicine, vol. 98, no. 13, 2019.

[19] P. Guilpain, C. L. Bihan, V. Foulongne, P. Taourel, and V. L. Moing, "Rituximab for granulomatosis with polyangiitis in the pandemic of covid-19: lessons from a case with severe pneumonia," Annals of the Rheumatic Diseases, annrheumdis, vol. 80 , no. $1,2020$.

[20] G. Zaharchuk and G. Davidzon, "Artificial intelligence for optimization and interpretation of PET/CT and PET/MR images," Seminars in Nuclear Medicine, vol. 51, no. 2, pp. 134-142, 2021.

[21] L. Fichera, G. Li-Destri, and N. Tuccitto, "Fluorescent nanoparticle-based internet of things," Nanoscale, vol. 12, no. 17, pp. 9817-9823, 2020.

[22] E. Hittinger and P. Jaramillo, "Internet of Things: energy boon or bane?," Science, vol. 364, no. 6438, pp. 326-328, 2019.

[23] A. M. Dimmitt, J. A. Pelz, M. E. Albertson et al., "Evaluation of adaptive statistical iterative reconstruction- $\mathrm{V}$ reconstruction algorithm vs filtered back projection in the detection of hypodense liver lesions: reader performance and preferences," Journal of Computer Assisted Tomography, vol. 43, no. 2, pp. 200-205, 2019.

[24] Z. Deng, Y. Hu, P. Yang et al., "Diagnosis and treatment of an acute severe pneumonia patient with COVID-19: case report," Journal of Medical Virology, vol. 92, no. 10, pp. 1728-1730, 2020.

[25] K. Cho, J. Chae, R. Y. Kwon, S. C. Bong, and K. S. Cho, “The application of the filtered backprojection algorithm to solar rotational tomography," Astrophysical Journal, vol. 895, no. $1,2020$.

[26] W. C. Chen, J. S. Niu, I. P. Liu et al., "Study of a palladium (Pd)/aluminum-doped zinc oxide (AZO) hydrogen sensor and the Kalman algorithm for Internet-of-Things (IoT) application," IEEE Transactions on Electron Devices, vol. 67, no. 10, pp. 4405-4412, 2020.

[27] J. Chauhan and P. Goswami, "An integrated metaheuristic technique-based energy aware clustering protocol for Internet of Things based smart classroom," Modern Physics Letters B, vol. 34, no. 22, 2020.

[28] V. Chang, V. M. Muñoz, and M. Ramachandran, "Emerging applications of internet of things, big data, security, and com- plexity: special issue on collaboration opportunity for IoTBDS and COMPLEXIS," Computing, vol. 102, no. 6, pp. 1301-1304, 2020.

[29] R. Ceipek, J. Hautz, A. de Massis, K. Matzler, and L. Ardito, "Digital transformation through exploratory and exploitative internet of things innovations: the impact of family management and technological diversification $*$," Journal of Product Innovation Management, vol. 38, no. 1, pp. 142-165, 2021.

[30] D. T. Braddock, P. R. Stabach, K. Zimmerman, D. Kavanagh, C. T. Sauei, and K. J. Yarema, "Protein engineering and glycan optimization improves pharmicokinetics of an enzyme biologic 10-fold," FASEB Journal, vol. 30, Supplement 1, 2019.

[31] R. Boudewijns, H. J. Thibaut, S. J. F. Kaptein et al., "STAT2 signaling restricts viral dissemination but drives severe pneumonia in SARS-CoV-2 infected hamsters," Nature Communications, vol. 11, no. 1, 2020.

[32] D. Blez, A. Soulier, F. Bonnet, E. Gayat, and M. Garnier, "Monitoring of high-flow nasal cannula for SARS-CoV-2 severe pneumonia: less is more, better look at respiratory rate," Intensive Care Medicine, vol. 46, no. 11, pp. 2094-2095, 2020.

[33] M. Beckmann, P. Maass, and J. Nickel, "Error analysis for filtered back projection reconstructions in Besov spaces," Inverse Problems, vol. 37, no. 1, 2020.

[34] O. Barker, "Realizing the promise of the Internet of Things in smart buildings," Computer, vol. 53, no. 2, pp. 76-79, 2020.

[35] A. Villa-Henriksen, G. T. C. Edwards, L. A. Pesonen, O. Green, and C. A. G. Sørensen, "Internet of Things in arable farming: implementation, applications, challenges and potential," Biosystems Engineering, vol. 191, pp. 60-84, 2020.

[36] T. Atefeh, P. Reza, S. K. Mojtaba, M. Asghar, R. Mehdi, and R. Reza, "Pediatric regional Drl assessment in common Ct examinations for medical exposure optimization in Tehran Iran," Radiation Protection Dosimetry, vol. 21, no. 3, pp. 563-574, 2020.

[37] M. A. Guillén, A. Llanes, B. Imbernón et al., "Performance evaluation of edge-computing platforms for the prediction of low temperatures in agriculture using deep learning," Journal of Supercomputing, vol. 77, no. 1, pp. 818-840, 2021.

[38] R. Du, Y. Liu, L. Liu, and W. Du, “A lightweight heterogeneous network clustering algorithm based on edge computing for 5G," Wireless Networks, vol. 26, no. 3, pp. 1631-1641, 2020. 